\title{
Progress in Treatment of Membranous Nephropathy with Traditional Chinese Medicine
}

\author{
Yingying Liu, Qi Jiang* \\ Department of Nephrology, China-Japan Union Hospital of Jilin University, Changchun, Jilin, 130033, \\ China \\ *corresponding author
}

Keywords: membranous nephropathy; traditional Chinese medicine; research progress; clinical value

\begin{abstract}
Membranous nephropathy is one of the common pathological types of nephrotic syndrome in China, which is difficult to heal. Western medicine uses immunosuppressants and cytotoxic hormones to treat this disease, causing many adverse reactions. The paper summarized ideas and experiences of treatment of membranous nephropathy with traditional Chinese medicine in recent years based on literature and clinical research collected by retrieving relative electronic materials and gained a lot of consensus. Literature review shows that there are many research on treatment of membranous nephropathy with traditional Chinese medicine whose curative effect is good and is worth learning. However, the development trend in recent years shows that the combination of traditional Chinese medicine and Western medicine has certain potential and advantages. There are many reports about the use of low-dose hormonal drugs and traditional Chinese medicine. The combination of traditional Chinese medicine and Western medicine for the treatment of membranous nephropathy is worthy of clinical promotion and application.
\end{abstract}

\section{Introduction}

Membranous nephropathy is a pathological diagnosis term, which is common in patients over 40 years old. It is difficult to treat this disease with insidious onset, complicated pathogenesis, high incidence and long course, and its characteristic pathological feature is diffused thickening of the basement membrane caused by many regular immune complex deposits on the epithelial side of the glomerular capillary, which can form "rete pegs". The clinical manifestation is nephrotic syndrome featured with a large amount of proteinuria, hypoalbuminemia, hyperlipidemia and edema. As the disease progresses, it often ends with chronic renal failure. MN can be divided into idiopathic membranous nephropathy (IMN) and secondary membranous nephropathy (SMN) according to the cause of the disease. IMN has no obvious inducements, accounting for about two-thirds of MN. SMN is caused by a variety of secondary factors (including viral infection, autoimmune diseases, malignant tumors, non-steroidal anti-inflammatory drugs, and familial and metabolic factors). Treatment of MN with the use of traditional Chinese medicine and Western medicine not only reduces the adverse reactions of Western medicine, but also improves the efficacy. This article summarized and analyzed the research progress of treatment of membranous nephropathy with traditional Chinese medicine. The specific research is as follows:

\section{The Etiology and Pathogenesis of Membranous Nephropathy in Traditional Chinese Medicine}

There is no disease called "membranous nephropathy" in Chinese medicine. Chinese medicine usually analyzes MN from the perspective of syndromes and MN is always related to "edema", "turbid urine", "pstranguria due to chyluria", "lumbago", "consumptive disease" and other diseases in traditional Chinese medicine. In the occurrence and development of membranous nephropathy, most doctors believe that $\mathrm{MN}$ is an asthenia in origin and in superficiality. Dysfunction of the kidney, spleen, lungs, bladder and liver is the main pathogenesis. Kidney deficiency is the basic 
cause. The dysfunction of organs has different role in this disease and the internal resistance of surgical diseases can also cause this disease. Surgical diseases include wind pathogen, cold or hot pathogen, sore toxin, blood stasis and so on. Chen Yiping believes that IMN has four characteristics of deficiency, dampness, phlegm and heat in the etiology and pathogenesis. Spleen and kidney deficiency is the basic pathogenesis of this disease. When the spleen becomes unhealthy, the body water will overflow into the skin to cause edema. The kidney deficiency will lose body water to cause edema; the spleen is to adjust dysfunction, so the spleen deficiency will lead to diarrhea; the kidney deficiency will cause loss of body water, leading to proteinuria. Liu Yuning believes that IMN spleen and kidney deficiency is the basic cause in the pathogenesis. The blood stasis in the whole body can lead to cold or hot pathogen, which is the main feature of this disease. In addition, liver stagnation cannot be ignored.

\section{Treatment of Membranous Nephropathy with Traditional Chinese Medicine}

\subsection{Dialectical treatment.}

With summarizing the experience of the instructor, a treatment plan based on expelling wind. dredging collat, benefiting the kidney and invigorating the circulation of blood has formed in the clinical practice of treating membranous nephropathy. It is believed that the spleen and kidney inefficiency, dampness retardarce and blood stasis are the main pathological parts of formation of membranous nephropathy proteinuria. To treat disease, medicine should be used for benefiting body and activating blood circulation to treat spleen and kidney inefficiency, dampness retardarce and blood stasis. The focus is to reduce proteinuria and stabilize renal function. The prescriptions are as follows: commonly used fresh astragalus membranaceus, eucommia, dogwood benefiting the kidney, coix seed, fried atractylodes and common yam rhizome benefiting the spleen and stomach; vine root, rhizoma dioscoreae nipponicae, silkworm and phlegm can be used to circulate blood and reduce dampness; when there is internal and external cold, it is necessary to treat the external cold first; when the internal cold is raging, it is best to search for the cause of the cold and cure it; pyrrosia lingua, radix ranunculi ternati and plantain seed can be used to stimulate the circulation of the blood and relax the muscle.

\subsection{Expert treatment.}

Chen Yiping divides membranous nephropathy into 3 syndrome types: 1) Guizhi Fuling Capsule can be used to treat membranous nephropathy with kidney deficiency, phlegm, body water stasis and stagnation; 2) Medicine called Chen's Qingre Membrane Kidney can be used to treat membranous nephropathy with kidney deficiency, dampness and heat to clear dampness and heat and promote blood circulation; 3) Medicine called Chen's Prescription benefiting Kidney Membrane can be used to clear dampness and descend the turbid. Zhang Lei uses astragalus membranaceus and rehmannia glutinosa decoction to treat membranous nephropathy. The decoction is filled with medicinal astragalus membranaceus, fresh rehmannia glutinosa, ripe rehmannia glutinosa, Chinese yam, dogwood, peony bark, rhizoma alismatis, the fruit of Chinese wolfberry and Poria cocos; Medicine called Sijunzi decoction can be used to treat spleen and kidney deficiency syndrome, which includes medicinal codonopsis pilosula, poria cocos, bighead atractylodes rhizome and honey-fried licorice root; Zuogui Pill can be used to treat liver and kidney deficiency syndrome, which contains fresh and ripe medicinal rehmannia glutinosa, Chinese yam, dogwood, peony bark, rhizoma alismatis, the fruit of Chinese wolfberry, poria cocos, deerhorn glue and turtle shell glue; Dabuyin Pill can be used to treat deficiency fire with inner heat, the medicine contains medicinal salt rhizoma anemarrhenae, salt golden cypress, ripe Rehmannia glutinosa and turtle shell; self-made Sanren decoction can be used to reduce dampness and turbidity, including medicinal coix seed, fructus amomi, almond, rhizoma pinellinae praeparata, polyporus umbellatus, poria cocos, cockroach, fried bighead atractylodes rhizome, rhizoma alismatis and cassia twig; to treat dampness and heat syndrome, medicinal fried jasmine, oriental wormwood, scutellaria barbata, portulaca oleracea and rhizoma alismatis should be used; to treat blood stasis syndrome, self-made 
blood activating and lipid-lowering medicine can be used, including medicinal ramuli euonymi, lotus leaf and rhizoma sparganii. On the basis of regulating spleen and stomach with reinforcing vital energy, Zou Yanqin emphasized the importance of expelling wind, dredging collat, promoting blood circulation, reducing dampness, extinguishing wind and resolving phlegm; to treat patients with the external wind (cold or wind-heat) which harms the kidney, medicine should be use to infiltrate the throat, containing radix scrophulariae, ophiopogon japonicus, honeysuckle, blackberry lily, platycodon grandiflorum, fresh liquorice, silkworm and periostracum cicada; to treat patients with severe heat toxin, it is necessary to add scutellaria baicalensis, jasmine, poria cocos, coix seed, plantain, radix ranunculi ternati and oldenlandia diffusa. On one hand, this will clear away heat and control pharyngitis; on the other hand, it will clear away damp heat to reduce kidney damage.

\subsection{Compound treatment.}

Wang Lin used traditional Chinese medicine of benefiting Qi (a term in traditional Chinese medicine, which is related to health) for activating blood circulation to treat membranous nephropathy. Compared with traditional Chinese medicine with immunosuppressive therapy, Chinese medicine used powder to treat membranous nephropathy. The drug composition includes codonopsis pilosula, rhizoma atractylodis macrocephalae, angelica sinensis, poria cocos and rhizoma atractylodis. For those with renal failure, it is necessary to add ligusticum wallichii, the root of kudzu vine and prepared rhubarb; for those who have edema, we need to add astragalus injection; for those with low proteinemia, we use black bean pills (black beans and astragalus membranaceus); for patients with blood stasis, we need to use capsule (mainly composed of leeches) to promote coronary circulation. It was concluded that medicine of benefiting Qi, activating blood circulation and reducing dampenss, has the advantages of stable curative effect and low recurrence rate in the treatment of membranous nephropathy. Jin Zhouhui treats membranous nephropathy based on medicine of promoting blood flow, invigorating spleen and soothing liver. The drug composition is codonopsis pilosula, radix astragali, the root of red-rooted salvia, curcuma zedoary, rhizoma curcumae, lumbricus, rhizoma atractylodis macrocephalae, Chinese yam and poria cocos. Spleen deficiency and damp heat syndrome is applied to clearing heat and dampness method, adding oldenlandia diffusa, scutellaria baicalensis, coix seed and pyrrosia lingua; spleen and kidney deficiency syndrome is applied to method of warming kidney and eliminating dampness, adding morinda officinalis, herba epimedii, Cistanche and motherwort. The treatment takes 6 months and the total effective rate is $92.98 \%$. Ma Chuanwu and other people treat 42 cases of refractory membranous nephropathy. The treatment group used medical soup (21 cases) of astragalus membranaceus and grifola, including medicinal jastragalus membranaceus, codonopsis pilosula, yam, coix seed, grifola, ligusticum wallichii, angelica sinensis, fresh ginger skin, motherwort, oldenlandia diffusa, herba epimedii and the root of red-rooted salvia; for patients with heat, it is necessary to add rehmannia, lalang grass rhizome and moutan bark; for patients with edema, we need to add pericarpium arecae, exocarpium benincasae and pyrrosia lingua; for patients with blood stasis, it is necessary to add leech, carthamus tinctorious and ground beeltle; for patients with yin deficiency, we can add Morinda officinalis, gorgon fruit, mantis egg-case and cinnamon. The control group (21 cases) was treated with hormone with mycophenolate mofetil and the course of treatment was 6 months. The treatment group always had efficiency of 95. 2\%, better than that of the control group 71.43\% $(\mathrm{P}<0.05)$. Chen Yiping used Shenqi granules with cyclophosphamide to treat patients with membranous nephropathy. After 48 weeks, the glomerular filtration rate increased $3 \mathrm{~mL} / \mathrm{min} / 1.73 \mathrm{~m}^{2}$ compared with that before treatment, better than the traditional Chinese medicine control group $(\mathrm{P}<0.05)$.

\subsection{Unit drug and effective component research.}

Ligustrazine protects renal function by reducing glomerular subcutaneous immune complex deposition, reducing podocyte injury and inhibiting glomerular basement membrane thickening; it can antagonize platelet aggregation and inhibit microthrombotic formation in the glomerulus; it can lower blood lipids and reduce glomerular sclerosis caused by mesangial proliferation; it can dilate blood vessels, advance renal microcirculation and improve endogenous creatinine clearance in mice 
with membranous nephropathy. Hematoxylon mainly contains flavonoids and sterols. It can be used to treat mice with membranous nephropathy with hematoxylin water extract, which can reduce proteinuria in experimental mice, increase plasma albumin level, increase renal blood flow, and delay glomerular sclerosis. Ethyl acetate extract of hematoxylon can inhibit mesangial cell proliferation, reduce immune complex formation, increase renal blood flow, increase glomerular filtration rate, improve microcirculation, and regulate renal tissue repair and regeneration of mice with membranous nephropathy. Hirudin can reduce glomerular fibrin-associated antigen deposition, reduce mesangial cell proliferation and glomerular sclerosis, reduce proteinuria, and improve renal function and hypoproteinemia. The main active ingredient of flos abelmoschus manihot is general flavonoids, which can inhibit immunity, reduce inflammation, improve renal fibrosis and protect renal tubular epithelial cells. It can reduce proteinuria and hematuria in clinical application. Astragalus membranaceus contains trace element selenium, flavonoids, saponins, polysaccharides and other components, which can improve protein metabolism disorders of glomerular diseases, increase plasma albumin levels, reduce proteinuria, improve renal fibrosis and protect kidney function. In blood reperfusion of diabetic nephropathy and kidney deficiency, it can prevent and treat injury. Paris polyphylla can protect the kidney of mice with membranous nephropathy, inhibit the expression of nuclear factor-kappa B (NF- $\mathrm{BB})$ protein and mRNA in renal tissue of mice with membranous nephropathy, inhibit the accumulation of fibronectin in renal tissue, and reduce extracellular matrix deposition in kidney tissue. Ttripterygium glycosides can inhibit T lymphocytes, regulate the pathological balance of immune cell subsets, correct immune disorders, reduce immune complexes in glomerular deposition, restore glomerular filtration membrane charge barrier function, improve filtration membrane permeability, lower proteinuria, increase plasma albumin, and maintain renal function. Triptolide inhibits immunity, reduces inflammation, antagonizes membrane attack complex-mediated podocyte injury and improves podocyte disease.

\subsection{Integrating Chinese and Western medicine treatment.}

In the treatment of primary membranous nephropathy, He Lingzhi generally uses Chinese medicine with glucocorticoids and immunosuppressive therapy. In he early stage, the high dose of hormones will inevitably consume body water and result in kidney deficiency. Therefore, treatment should focus on benefiting kidney and reducing fire, and pill of six ingredients with rehmannia can be used. When the hormone reduces to a certain extent and the body is still dependent on hormones, the treatment should use Youguiwan and Erxian decoction. Director Meng Xiangzhen divided MN into spleen deficiency, dampness and heat accumulation, kidney deficiency, Qi deficiency, phlegm and blood stasis, spleen and kidney decay and dampness. We can treat this disease with the combination of traditional Chinese and Western medicine, develop a treatment method of hormone + immunosuppressive agent + empirical formula (benefiting Qi and reducing dampness) to promote the efficiency and reduce dampness with good effect.

\section{Summary}

In recent years, a large number of clinical studies in China have shown that the combination of traditional Chinese and Western medicine has achieved remarkable results in the treatment of IMN, which not only reduces the adverse reactions of Western medicine and the recurrence rate of diseases, but also improves the overall treatment effect. It is envisaged that in the future, on the basis of extensively conducting renal biopsy techniques, the TCM syndrome differentiation and evaluation standards will be developed early, and a more effective treatment plan integrating Chinese and Western medicine will be explored to give full play to the advantages of Chinese and Western medicine in the treatment of membranous nephropathy.

\section{References}

[1] She A'min, Sheng Meixiao. Progress in Traditional Chinese Medicine Research on Membranous Nephropathy [J]. Journal of Changchun University of Traditional Chinese Medicine, 2016, 32(01): 
205-209.

[2] Tang Long, Zhang Shengrong. A Research on the Status of TCM Syndrome Treatment of Idiopathic Membranous Nephropathy [J]. Global Chinese Medicine, 2016, 9(02): 253-256.

[3] Lu Shilong, Wang Longlong and Huang Guodong. Research progress in the Treatment of Membranous Nephropathy with Traditional Chinese Medicine [J]. Journal of Guangxi University of Chinese Medicine, 2016, 19(02): 84-87.

[4] Yu Siming, Liu Haixia, Guo Dandan and Qi Chunpeng. Progress in Treatment of Idiopathic Membranous Nephropathy with Traditional Chinese Medicine [J]. Chinese Journal of Clinical Healthcare, 2016,19(04):445-447 